\title{
Long Noncoding RNA Expression Profiling in Normal B-Cell Subsets and Hodgkin Lymphoma Reveals Hodgkin and Reed-Sternberg Cell-Specific Long Noncoding RNAs
}

Mina Masoumeh Tayari, ${ }^{*}$ Melanie Winkle, ${ }^{*}$ Gertrud Kortman, ${ }^{*}$ Jantine Sietzema, ${ }^{*}$ Debora de Jong, ${ }^{*}$ Martijn Terpstra, ${ }^{\dagger}$ Pieter Mestdagh, ${ }^{\ddagger}$ Frans G.M. Kroese, ${ }^{\S}$ Lydia Visser, ${ }^{*}$ Arjan Diepstra, ${ }^{*}$ Klaas Kok, ${ }^{\dagger}$ Anke van den Berg, ${ }^{*}$ and Joost Kluiver*

From the Departments of Pathology and Medical Biology, ${ }^{*}$ Genetics, ${ }^{\dagger}$ and Rheumatology and Clinical Immunology, ${ }^{\S}$ University of Groningen, University Medical Center Groningen, Groningen, the Netherlands; and the Center for Medical Genetics, ${ }^{\ddagger}$ Ghent University, Ghent, Belgium

Accepted for publication

May 18, 2016.

Address correspondence to Joost Kluiver, Ph.D.,

Department of Pathology and Medical Biology, University of Groningen, University Medical Center Groningen, Hanzeplein 1, 9700RB Groningen, the Netherlands. E-mail: j.l kluiver@umcg.nl.

\begin{abstract}
Hodgkin lymphoma ( $\mathrm{HL}$ ) is a malignancy of germinal center (GC) B-cell origin. To explore the role of long noncoding RNAs (IncRNAs) in $\mathrm{HL}$, we studied IncRNA expression patterns in normal B-cell subsets, $\mathrm{HL}$ cell lines, and tissues. Naive and memory B cells showed a highly similar IncRNA expression pattern, distinct from GC-B cells. Significant differential expression between HL and normal GC-B cells was observed for 475 IncRNA loci. For two validated IncRNAs, an enhanced expression was observed in $\mathrm{HL}$, diffuse large B-cell lymphoma, and lymphoblastoid cell lines. For a third IncRNA, increased expression levels were observed in $\mathrm{HL}$ and part of Burkitt lymphoma cell lines. RNA fluorescence in situ hybridization on primary $\mathrm{HL}$ tissues revealed a tumor cell-specific expression pattern for all three IncRNAs. A potential cis-regulatory role was observed for 107 differentially expressed IncRNA-mRNA pairs localizing within a $60-\mathrm{kb}$ region. Consistent with a cis-acting role, we showed a preferential nuclear localization for two selected candidates. Thus, we showed dynamic IncRNA expression changes during the transit of normal B cells through the GC reaction and widely deregulated IncRNA expression patterns in $\mathrm{HL}$. Three IncRNAs showed a tumor cell-specific expression pattern in $\mathrm{HL}$ tissues and might therefore be of value as a biomarker. (Am J Pathol 2016, 186: 2462-2472; http://dx.doi.org/10.1016/j.ajpath.2016.05.011)
\end{abstract}

Hodgkin lymphoma (HL) is a B-cell neoplasm characterized by a minority of neoplastic cells within an extensive inflammatory background. The incidence of HL is approximately three per 100,000 per year in Western countries, and it is most common in adolescents and young adults. ${ }^{1} \mathrm{HL}$ has been categorized into two disease entities, classic Hodgkin lymphoma (cHL) and nodular lymphocyte predominant Hodgkin lymphoma (NLPHL). cHL accounts for approximately $95 \%$ of all cases, whereas NLPHL is less common. The neoplastic cells of cHL [ie, Hodgkin and Reed-Sternberg (HRS) cells] originate from germinal center (GC) B cells. The HRS cells show a loss of B-cell phenotype with no or strongly reduced expression levels of the B-cell receptor, common B-cell markers, and transcription factors. ${ }^{2,3}$ In contrast, the neoplastic cells of NLPHL (ie, the lymphocyte predominant cells) have retained their B-cell phenotype. ${ }^{4}$ HRS cells can actively influence their microenvironment through the production of various cytokines and a variety of cell surface receptors. Among the factors and pathogens that contribute to $\mathrm{cHL}$ pathogenesis are NF-kB and Epstein-Barr virus. ${ }^{5} \mathrm{NF}-\kappa \mathrm{B}$ signaling efficiently activates transcription of a variety of genes, which are involved in survival, proliferation, and inflammation. ${ }^{6}$

The impact of noncoding (nc) RNAs on hematological malignancies, including $\mathrm{HL}$, has been well described for miRNAs. ${ }^{7}$ Next to miRNAs, another class of ncRNAs [ie, the

Supported by University Medical Center Groningen grants UMCG611006 (M.T.) and UMCG-613001 (M.W.) and Pediatric Oncology Foundation Groningen grant SKOG 14-001 (A.v.d.B., K.K., and J.K.).

Disclosures: None declared. 
long (l) ncRNAs] have recently received a lot of attention as important regulators of gene expression. ${ }^{8}$ lncRNAs are defined as transcripts $>200$ nucleotides in length that lack proteincoding potential. They are mostly categorized on the basis of their genomic location and orientation compared to proteincoding genes (eg, sense, antisense, intronic, or intergenic). ${ }^{9}$ Previously, a subset of IncRNAs has been shown to either positively ${ }^{10}$ or negatively ${ }^{11}$ regulate neighboring proteincoding genes (cis-acting lncRNAs). ${ }^{12}$ For instance, some nuclear IncRNAs were shown to function as transcriptional regulators of protein-coding genes in cis. This mechanism involves three-dimensional folding of chromatin juxtaposing regulatory sequences located several kilobases apart into close spatial proximity. ${ }^{12}$ There is increasing evidence that alterations in the expression levels of IncRNAs are linked to tumorigenesis. ${ }^{13,14}$ It is currently unknown to what extent lncRNAs are regulated in $\mathrm{B}$ cells during the $\mathrm{GC}$ reaction and possibly deregulated in HL. In this study, we generated lncRNA expression profiles of normal mature B-cell subsets and HL cell lines. RNA fluorescence in situ hybridization (FISH) was used to confirm expression in tumor cells of primary cHL tissues. Finally, we identified putative $c i s$-regulatory lncRNA bases on differential expression patterns of nearby protein-coding genes.

\section{Materials and Methods}

\section{Tissue Samples and Cell Lines}

Five formalin-fixed, paraffin-embedded cHL tissue samples were randomly selected from the Pathology files of the University Medical Center Groningen tissue bank. All five cHL cases were young adults (aged 20 to 30 years), four were of the nodular sclerosis subtype and one was of the mixed cellularity subtype according to World Health Organization classification. ${ }^{15}$ Epstein-Barr encoding region in situ hybridization revealed that one of the NS cases was Epstein-Barr virus positive. Normal B-cell subsets were sorted by fluorescenceactivated cell sorter from three different tonsil samples as described previously. ${ }^{16}$ The procedures were according to the guidelines of the medical ethics board of the University Medical Center Groningen. Written informed consent was obtained for the use of the tonsil samples from the parents of the children. The $\mathrm{HL}$ cell lines were cultured at $37^{\circ} \mathrm{C}$ under an atmosphere containing 5\% $\mathrm{CO}_{2}$ in RPMI-1640 medium (Cambrex Biosciences, Walkersville, MD) supplemented with $2 \mathrm{mmol} / \mathrm{L}$ ultraglutamine, $100 \mathrm{U} / \mathrm{mL}$ penicillin, $0.1 \mathrm{mg} / \mathrm{mL}$ streptomycin (Cambrex Biosciences), and 20\% (DEV, L540, SUP-HD1), 10\% (L1236, KM-H2), or 5\% (L428) fetal calf serum (Cambrex Biosciences). We routinely confirmed the identity of our cell lines using the PowerPlex 16 HS System (Promega, Leiden, the Netherlands).

\section{RNA Isolation from Cell Lines, B-Cell Subsets, and Nuclear and Cytoplasmic Fractions}

RNA was isolated from cell lines and B-cell subsets as described earlier. ${ }^{17}$ RNA concentration was measured with a
NanoDrop 1000 Spectrophotometer (Thermo Fisher Scientific Inc., Waltham, MA), and integrity was assessed by analysis of the $18 \mathrm{~S} / 28 \mathrm{~S}$ bands on a $1 \%$ agarose gel. Nuclear and cytoplasmic fractions were isolated from the cell lines L1236, L428, and L540 as described previously. ${ }^{17}$

\section{Microarray Analysis}

Design of the custom IncRNA microarray, labeling and hybridization procedures, data analysis, and generation of heat maps were performed as described previously. ${ }^{17}$ Naive, memory, GC-B, and HL cell lines were Cy3 labeled using 50 to $100 \mathrm{ng}$ total RNA. Probes consistently flagged as present and expressed in the 10 to 100th percentile in at least one of the three conditions for the comparison of naive, GC-B, and memory B cells were included in the statistical analyses. For the comparison of the GC-B and HL samples, probes that consistently expressed one of two conditions were included. We detected 10,469 1ncRNA and 17,885 mRNA probes with consistent signals above the background for the three B-cell subsets and 9955 lncRNA and 17,551 mRNA probes for the HL versus GC-B cell comparison. Significant differences in transcript abundance were determined by one-way analysis of variance (normal B-cell subsets) or unpaired $t$-test (HL versus GC-B cells). Benjamini-Hochberg multiple testing correction was applied. Microarray data used for this publication were deposited at the National Center for Biotechnology Information Gene Expression Omnibus (http://www.ncbi.nlm. nih.gov/geo; accession number GSE81086).

\section{Quantitative RT-PCR}

First-strand cDNA was synthesized with 500 ng RNA in a total reaction volume of $20 \mu \mathrm{L}$ using the following reagents: random hexamer primers, $10 \mathrm{mmol} / \mathrm{L}$ dNTP mix, $5 \times$ firststrand buffer, $0.1 \mathrm{~mol} / \mathrm{L}$ dithiothreitol solution, $1 \mu \mathrm{L}$ RNaseOUT Recombinant Ribonuclease Inhibitor, and $1 \mu \mathrm{L}$ Superscript II Reverse Transcriptase (Life Technologies). PCRs were performed in triplicate on a Lightcycler 480 system (Roche, Penzberg, Germany). Each quantitive PCR (qPCR) contained 1 ng cDNA, SYBR Green Master Mix (Applied Biosystems), and $3 \mu \mathrm{mol} / \mathrm{L}$ primers in a reaction volume of $10 \mu \mathrm{L}$. U6 served as endogenous control for normalization. Relative expression levels are calculated as $2-\Delta \mathrm{Ct}$. For analysis of the subcellular localization, expression was normalized to $18 \mathrm{~S}$ when the cytoplasmic fraction was compared to the total fraction and to U3 small nucleolar RNA when the nuclear fraction was compared to the total fraction. Table 1 contains the sequences of all primers used in this study.

\section{Processing of The Cancer Genome Atlas RNA-Seq Data}

Fastq files for 20 randomly selected samples per cancer type were mapped to the human genome (build hg19) using tophat. Gene expression values were quantified using 
Table 1 Sequences of the Primers Used in This Study

\begin{tabular}{|c|c|}
\hline Transcripts & Sequence \\
\hline \multirow{2}{*}{$\begin{array}{l}\text { FLJ42351 } \\
\text { (TCONS_00002672) }\end{array}$} & F: 5'-TTGTGGCTCATGCCATATGAA-3' \\
\hline & R: $5^{\prime}$-CAAAGATCCTGTGGGCAGTCA-3' \\
\hline \multirow{2}{*}{$\begin{array}{l}\text { LINC00116 } \\
\quad \text { (TCONS_00003368) }\end{array}$} & F: 5'-CATGGCCGGCTCTTCCT-3' \\
\hline & R: $5^{\prime}$-TCATAAAGTGCAAGAAGAAGTCATTTC-3' \\
\hline \multirow[t]{2}{*}{ LINC00461 } & F: 5'-CTTAAGCGCGGCAAGTATCC-3' \\
\hline & R: $5^{\prime}$-GCCAGACTCCAGGTCCTGATC-3' \\
\hline \multirow[t]{2}{*}{ TCONS_12_00015489 } & F: 5'-GGCTGCAGATGGCAGGATT-3' \\
\hline & R: 5'-TGCTGTACAGATACACCACGGAAT-3' \\
\hline \multirow[t]{2}{*}{ TCONS_00002977 } & F: 5'-AAGCAGATGCTGTGCCTGATAC-3' \\
\hline & R: 5'-TTCTCGACCCAGAAGCTCAAG-3' \\
\hline \multirow[t]{2}{*}{ TCONS_00021708 } & F: $5^{\prime}-T G G A T T T T A C A G G C C C T C T T C A-3^{\prime}$ \\
\hline & R: 5'-GCTCCTGCCTCTGTTTTGCT-3' \\
\hline \multirow[t]{2}{*}{ TCONS_00025860 } & F: $5^{\prime}$-CGCGATTTGCAGGATTCC-3' \\
\hline & R: $5^{\prime}-$ CAAATGTGGGCACTTAAAAGCA-3' \\
\hline \multirow[t]{2}{*}{ TCONS_00007500 } & F: 5'-TGGGTTAACACTGCTTTTATGAGTTG-3' \\
\hline & R: $5^{\prime}$-GCTGGCTCAGGAGTGAAGCT-3' \\
\hline \multirow[t]{2}{*}{ TCONS_12_00030240 } & F: $5^{\prime}-$ CACACTCCAAGGAAACGCAA- $3^{\prime}$ \\
\hline & R: $5^{\prime}$-GGGATGACTGACCTCCTCTACC-3' \\
\hline \multirow[t]{2}{*}{ TCONS_00029175 } & F: 5'-AGTTCATTCAACTGGTGATCTTAAGC-3' \\
\hline & R: 5'-GCTGAGTCTACCTGGAGTCCATTATT-3' \\
\hline \multirow[t]{2}{*}{ TCONS_L2_00009699 } & F: 5'-GCTATTTTAAAAGGGTGTCCAG-3' \\
\hline & R: 5'-CTGTACTAAGCCTCCCCCAGACT-3' \\
\hline \multirow[t]{2}{*}{ TCONS_12_00012111 } & F: 5'-CTAAACCTCCTGCAAAAGTGGAA-3' \\
\hline & R: 5'-TGTTTGCACTTTTTTGTCTGAAGAT-3' \\
\hline \multirow[t]{2}{*}{ U6 } & F: $5^{\prime}-$ TGGAACGATACAGAGAAGATTAGCA-3' \\
\hline & R: 5'-AAAATATGGAACGCTTCACGAATT-3' \\
\hline \multirow[t]{2}{*}{ RPPH1 } & F: $5^{\prime}$-AGCTTGGAACAGACTCACGG-3' \\
\hline & R: $5^{\prime}$-AATGGGCGGAGGAGAGTAGT-3' \\
\hline \multirow[t]{2}{*}{ DANCR } & F: 5'-CGTCTCTTACGTCTGCGGAA-3' \\
\hline & R: 5'-TGGCTTGTGCCTGTAGTTGT-3' \\
\hline \multirow[t]{2}{*}{ MT-TK } & F: 5'-CGGCTAGCTCAGTCGGTAGA-3' \\
\hline & R: $5^{\prime}-$ CCAACGTGGGGCTCGAAC- $3^{\prime}$ \\
\hline \multirow[t]{2}{*}{ MIAT } & F: 5'-TGGAGGCATCTGTCCACCCATGT-3' \\
\hline & R: $5^{\prime}-$ CCCTGTGATGCCGACGGGGT-3' \\
\hline \multirow[t]{2}{*}{ ANRIL } & F: 5'-AAGCCGCTCCGCTCCTCTTCT-3' \\
\hline & R: $5^{\prime}$-GCCGTGTCCAGATGTCGCGT-3' \\
\hline \multirow[t]{2}{*}{ XIST } & F: $5^{\prime}$-GTCCTTTCTTTTGACCCCAGAA-3' \\
\hline & R: 5'-GAGCCTGGCACTTTTTTTTCC-3' \\
\hline \multirow[t]{2}{*}{$18 \mathrm{~S}$} & F: 5'-CGGCTACCACATCCAAGGA-3' \\
\hline & R: 5'-CCAATTACAGGGCCTCGAAA-3' \\
\hline \multirow[t]{2}{*}{ U3 } & F: 5'-AACCCCGAGGAAGAGAGGTA-3' \\
\hline & R: $5^{\prime}$-CACTCCCCAATACGGAGAGA-3' \\
\hline \multirow[t]{2}{*}{ MEF2C } & F: $5^{\prime}-$ CAAATGCAGGGCCCCTT- $3^{\prime}$ \\
\hline & R: $5^{\prime}$-GATATGCACTTACTGAATTCCA-3' \\
\hline \multirow[t]{2}{*}{ SLC20A1 } & F: $5^{\prime}-\mathrm{CTGGCTCCGGTCCAAGAA-3^{ \prime }}$ \\
\hline & R: $5^{\prime}-T G T G A C A A A C C A G G C C A T A A A A-3^{\prime}$ \\
\hline
\end{tabular}

$F$, forward; $R$, reverse.

Cufflinks software version 2.1.1 (http://cole-trapnell-lab. github.io/cufflinks/releases/v2.1.1) on the basis of the Ensembl reference transcriptome annotation (version 75).

\section{Immunohistochemistry and RNA-FISH}

An immunohistochemical staining for chemokine ligand (CCL) 17 was performed as described previously ${ }^{18}$ to confirm that the tumor cells were positive for CCL17. FISH probe sets and reagents (QuantiGene ViewRNA ISH Tissue 2-Plex Assay kit) were purchased from Affymetrix (Santa Clara, CA). RNA-FISH was performed according to the instructions provided by the manufacturer using formalin-fixed, paraffin-embedded tissue sections ( $5 \mu \mathrm{m}$ thick). A probe set for CCL17 was used as a positive control and to identify the HRS tumor cells in combination with one of the three probe sets for the selected lncRNAs. CCL17 was visualized with a type 6 probe set (Fast Blue) and the lncRNAs were visualized with type 1 probe sets (Fast Red). Custom probe sets designed by the manufacturer covered the exons that are common to all isoforms. Slides were imaged on a Leica SP8 confocal microscope.

\section{Identification of Differentially Expressed IncRNA-mRNA Pairs Mapping within a 60-kb Region}

To identify putative cis-acting lncRNAs, we determined for each differentially expressed IncRNA whether there was a differentially expressed protein-coding gene in close vicinity (directly adjacent) using Galaxy (https://usegalaxy.org; last accessed May 13, 2016). ${ }^{19,20}$ The genomic coordinates of all differentially expressed probes were uploaded using the get data tool. We next defined the neighboring differentially expressed mRNAs using operate on genomic intervals tools, fetch closest nonoverlapping feature for every interval and joint data sets. The resulting data set was subsequently filtered for IncRNA-mRNA pairs within $60 \mathrm{~kb}$ of each other.

\section{Results}

Highly Dynamic IncRNA Expression Changes during the Germinal Center Reaction

Statistical analysis of the gene expression profiles generated for naive, GC, and memory B cells revealed 401 significantly differentially expressed lncRNA probes (251 lncRNA loci) (analysis of variance, false discovery rate $<0.05$ ) with a fold-change of at least 2 in expression levels. The naive and memory B-cell subsets showed a highly similar expression pattern with a significant differential expression for only two probes. In contrast, GC-B cells showed a distinct lncRNA expression pattern with 377 differentially expressed probes compared to naive and 376 compared to memory B cells. Unsupervised hierarchical clustering using the 401 lncRNA probes revealed two distinct clusters, one cluster with the GC-B cell samples and a second cluster with the naive and memory B-cell subsets (Figure 1A). Analysis of the differentially expressed mRNAs (2908 probes, 2505 loci) revealed a clustering pattern similar to that of lncRNAs (Supplemental Figure S1A).

Next, we clustered the six HL cell lines together with the B-cell subsets using the 401 lncRNA probes that were significantly different between the three normal B-cell subsets. This revealed a pairwise clustering of the GC-B cells and HL cell lines in one tree and the naive and memory B-cells in a separate tree (Figure 1B). The NLPHL cell line DEV clustered in the same branch as the GC-B cells, whereas the five cHL cell lines clustered in a separate 
A

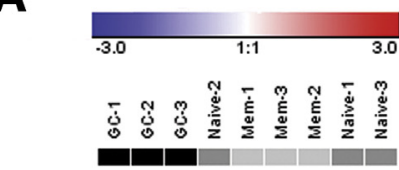

B

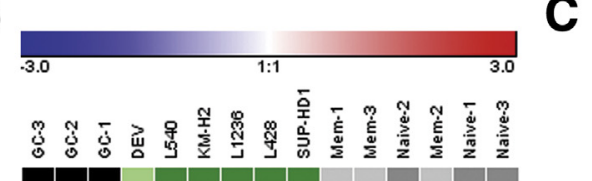

C

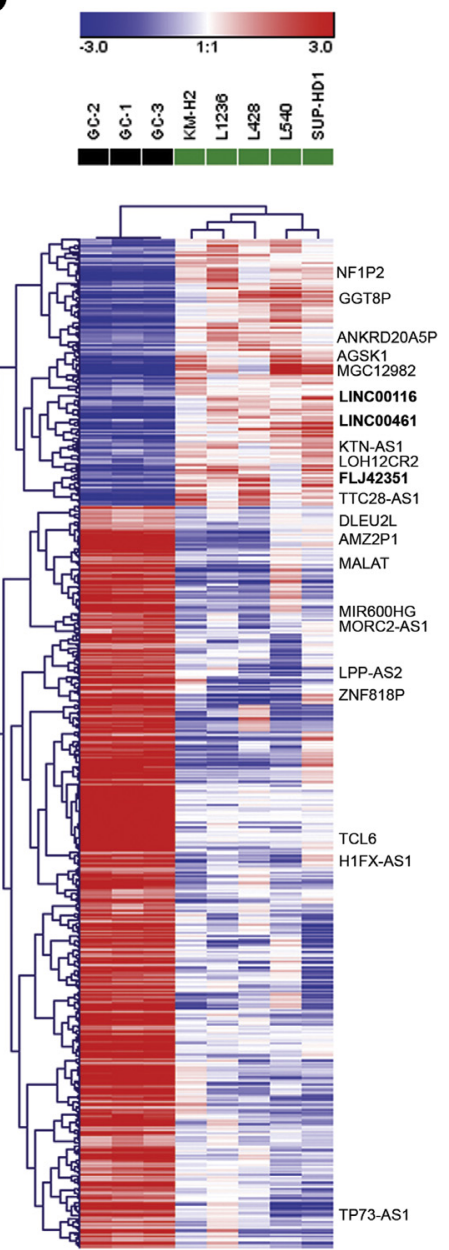

Figure 1 Unsupervised hierarchical clustering of differentially expressed IncRNAs. A: Heat map of the 401 IncRNA probes (251 lncRNA loci) differentially expressed between germinal center (GC), naïve, and memory (Mem) B cells. B: Heat map of the same 401 human IncRNA probes, including the cHL cell lines L540, KM-H2, L1236, L428, and SUP-HD1 and the NLPHL cell line DEV. C: Unsupervised clustering of the 639 IncRNA probes (475 IncRNA loci) differentially expressed between $\mathrm{CHL}$ and GC-B cells. The positions of some known and well-annotated IncRNAs are indicated in the heat maps. In bold are the three IncRNA candidates that were further studied by RNA fluorescence in situ hybridization.

branch next to the GC-B cells. This is consistent with the GC-B cell origin of the tumor cells of HL, with a loss-of-Bcell phenotype in cHL but not in NLPHL. A similar result was observed using the 2908 mRNA probes that were differentially expressed between the normal B-cell subsets (Supplemental Figure S1B).

\section{IncRNA Expression Is Widely Deregulated in $\mathrm{CHL}$}

Comparison of the expression profiles of $\mathrm{cHL}$ (excluding the NLPHL cell line DEV) to GC-B cells revealed a significantly differential expression for 639 lncRNA probes (475 lncRNA loci) (Supplemental Table S1 and Figure 1C). ${ }^{21}$ Comparison of the mRNA expression profiles revealed 2402 differentially expressed probes (2023 loci) (Supplemental Figure S1C). IncRNAs were predominantly down-regulated in HL (74\% down-regulated versus $26 \%$ up-regulated), whereas mRNAs showed a more equal distribution between up-regulated and down-regulated transcripts $(56 \%$ down-regulated versus $44 \%$ upregulated). Comparison of the lncRNAs and mRNAs differentially expressed between the normal B-cell subsets and between cHL cell lines and GC-B cells revealed a limited overlap of 70 lncRNA and 581 mRNA probes.

To validate the differential expression patterns, we designed RT-qPCR primer sets for nine up-regulated and five down-regulated lncRNAs randomly selected from the set of IncRNAs differentially expressed between cHL and GC-B cells. All nine up-regulated lncRNAs showed a higher expression in cHL consistent with the microarray data, with significant differences for six of them (Supplemental Figure S2). For the down-regulated lncRNAs, we could confirm significant changes for three of five lncRNAs. For the remaining two lncRNAs, we were not able to design primer sets that could amplify these lncRNA transcripts. 
IncRNA Expression in B Cells, Lymphoma Cell Lines, and Primary $\mathrm{CHL}$ Tissues

We selected three of the validated up-regulated IncRNAs (ie, FLJ42351, LINC00116, and LINC00461) (Supplemental Figure S3) for further expression analysis in normal B-cell subsets and a panel of lymphoma cell lines. This revealed high levels in HL, diffuse large B-cell lymphoma (DLBCL), and lymphoblastoid cell lines (LCL) for FLJ42351 and LINC00461 (Figure 2, A and C). For LINC00116, high levels were mainly observed in the HL cell lines and in part of the Burkitt lymphoma cell lines (Figure 2B). Reanalysis of The Cancer Genome Atlas (TCGA) RNA-seq data across 24 different cancer types revealed relative low expression levels for FLJ42351 and LINC00116 without clear differences between different cancer tissues (Figure 2). For LINC00461, higher levels were observed only for lower-grade glioma.

Next, we used RNA-FISH to study the expression of the three in cHL cell lines up-regulated lncRNAs in tissue sections of primary $\mathrm{cHL}$ cases. The five randomly selected cHL cases showed positive staining for CCL17 by immunohistochemistry (data not shown). RNA-FISH using the CCL17 probe set as a positive control revealed staining of the HRS cells in four of the five cases. RNA-FISH using the lncRNA probe sets revealed a tumor cell-specific staining in the four remaining cHL cases for all three lncRNAs (Figure 3). The probe sets for FLJ42351 and LINC00461
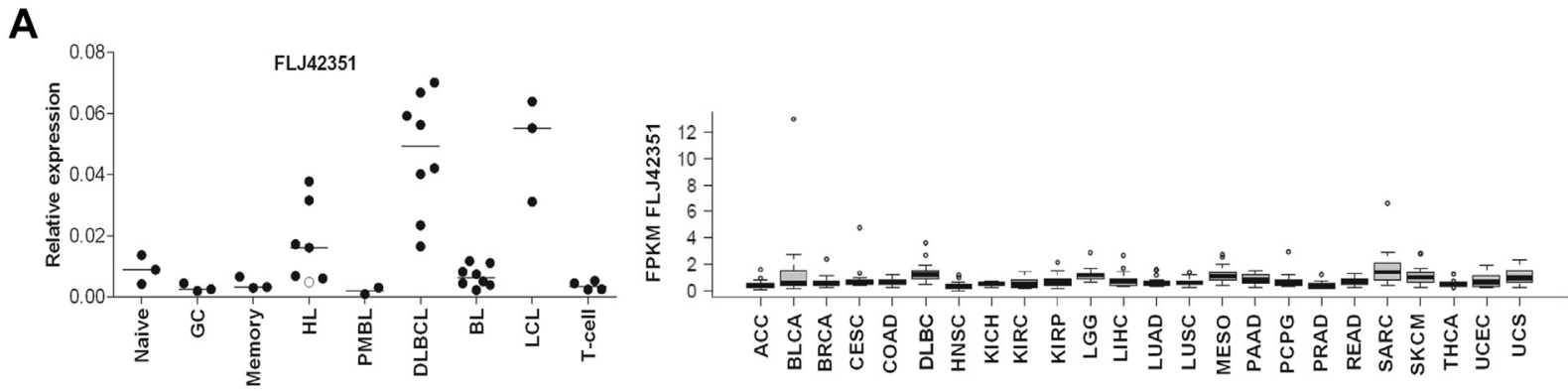

B
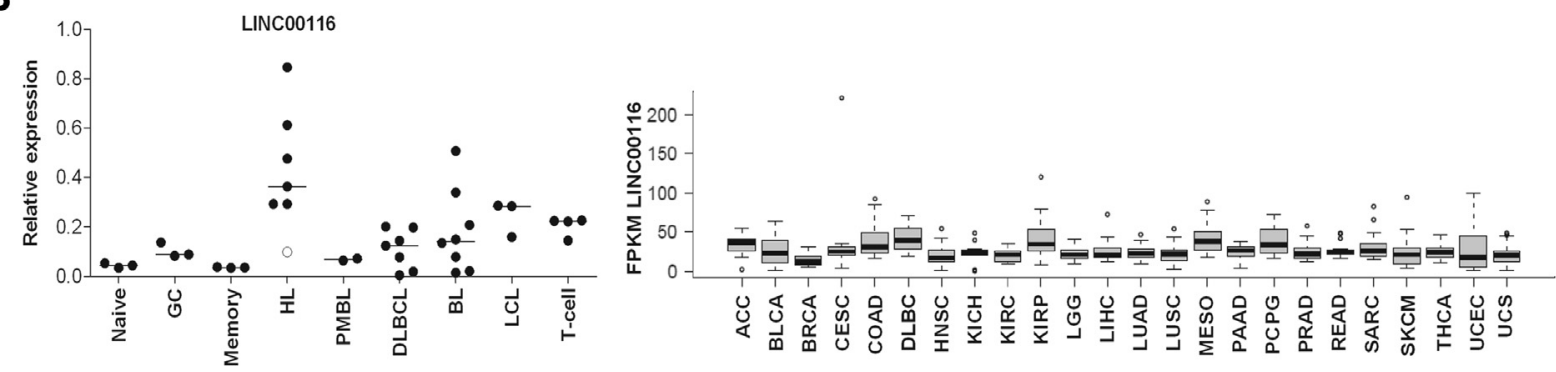

C
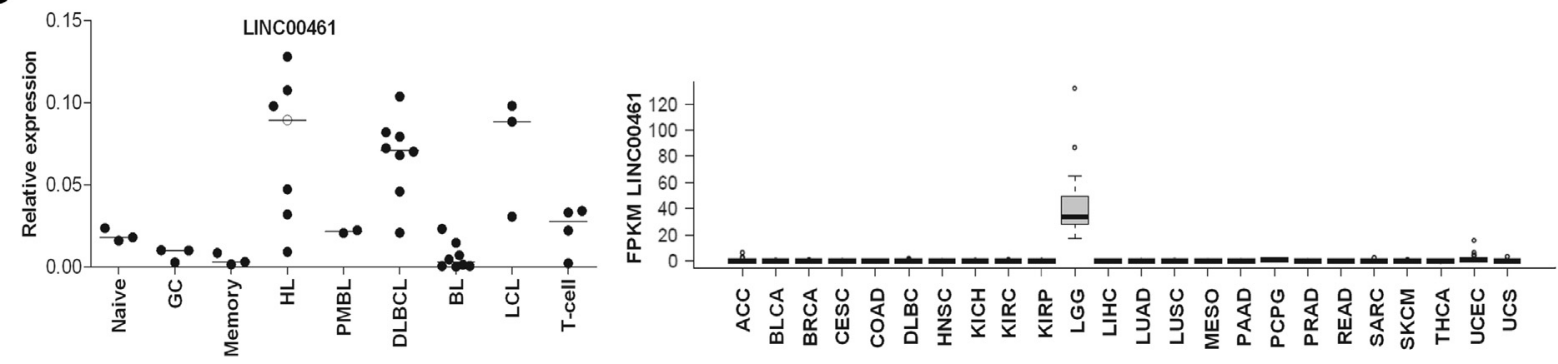

Figure 2 IncRNA expression levels in normal B cells, lymphoma, and other cancer tissues. Quantitative RT-PCR analysis and reanalysis of The Cancer Genome Atlas RNA-seq data for FLJ42531 (A), LINC00116 (B), and LINC00461 (C). Groups consisted of: Naive, CD19 IgD ${ }^{+}$CD38 ${ }^{-}$cells (3); GCB, $\mathrm{CD} 19^{+} \mathrm{IgD}^{-} \mathrm{CD}_{3} 8^{+}$(3); memory, $\mathrm{CD}_{19}^{+} \mathrm{IgD}^{-} \mathrm{CD} 38^{-}$(3); HL, L428, L540, L1236, KM-H2, L591, SUP-HD1, and NLPHL, DEV (open circle); PMBL, K1106P, and MEDB1; DLBCL, Karpas 422, SU-DHL-4, SU-DHL-5, SU-DHL-6, SU-DHL-10, OCILy-3, U-2932, DOHH2; BL, ST486, DG75, RAM0S, CA46, BL65, RAJI, Jiyoye, and Nawalma; LCL, LCL6A, LCL39, LCL89; T-cell, Jurkat, HUT-78, KARPAS 299, SR678. ACC, adrenocortical carcinoma; BL, Burkitt lymphoma; BLCA, bladder urothelial carcinoma; BRCA, breast invasive carcinoma; CESC, cervical squamous cell carcinoma and endocervical adenocarcinoma; COAD, colon adenocarcinoma; DLBCL, diffuse large B-cell lymphoma; GC, germinal center B cells; HL, Hodgkin lymphoma; HNSC, head and neck squamous cell carcinoma; KICH, kidney chromophobe; KIRC, kidney renal clear cell carcinoma; KIRP, kidney renal papillary cell carcinoma; LCL, lymphoblastoid cell line; LGG, brain lower grade glioma; LIHC, liver hepatocellular carcinoma; LUAD, lung adenocarcinoma; LUSC, lung squamous cell carcinoma; MES0, mesothelioma; NLPHL, nodular lymphocyte predominant Hodgkin lymphoma; PAAD, pancreatic adenocarcinoma; PCPG, pheochromocytoma and paraganglioma; PMBL, primary mediastinal B-cell lymphoma; READ, rectum adenocarcinoma; SARC, sarcoma; SKCM, skin cutaneous melanoma; T-cell, T-cell leukemia and lymphoma cell lines; THCA, thyroid carcinoma; UCEC, uterine corpus endometrial carcinoma; UCS, uterine carcinoma. 

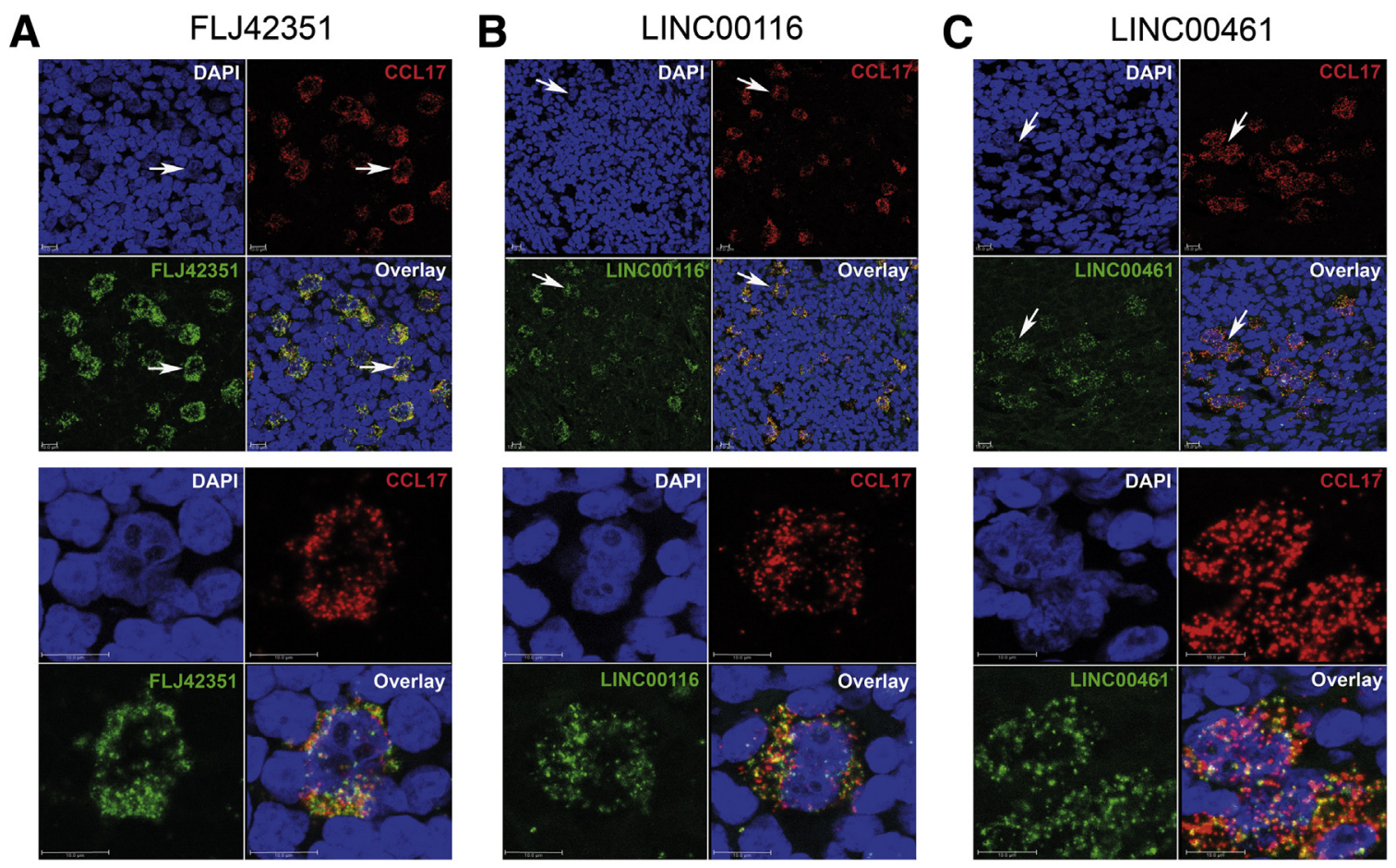

Figure 3 Expression of FLJ42351, LINC00116, and LINC00416 is restricted to the Hodgkin and Reed-Sternberg (HRS) cells of cHL cases. Dual-color RNA fluorescence in situ hybridization was performed using a probe set for chemokine ligand (CCL) 17 (tumor cell specifically expressed) in combination with probe sets for FLJ42351 (A), LINC00116 (B), or LINC00146 (C). Shown are representative images of the DAPI, CCL17 (in red, fast blue), and IncRNA (in green, Cy3/ fast red) signals and the overlay of the three. Arrows in the top row panels indicate the HRS tumor cell that was used for the magnified images that are shown in the bottom row panels. Scale bar $=10 \mu \mathrm{m}$.

showed positive signals in three cases and weak positive signals in one case, the probe set for LINC00116 showed positive signals in all four cases.

\section{IncRNAs and Neighboring Protein-Coding Genes}

To identify lncRNAs that potentially affect the expression of nearby protein-coding genes, we determined which of the differentially expressed lncRNAs was close to a differentially expressed mRNA. Within the 251 lncRNAs differentially expressed between the normal B-cell subsets, we identified 51 putative cis-regulatory IncRNAs with a probe-to-probe distance of up to $60 \mathrm{~kb}$ to the differentially expressed mRNA (Supplemental Table S2). Of the 475 lncRNAs differentially expressed between $\mathrm{cHL}$ and GC-B cells, 59 were in close vicinity of a differentially expressed mRNA (Table 2).

Three of the putative cis-regulated IncRNA-mRNA pairs were detected in both the normal B-cell subsets and the HL versus GC-B analysis. For all three pairs, a positive correlation between the IncRNA and mRNA expression was observed in both analyses.

To support a putative cis-regulatory role for two (LINC00461 and FLJ42351) of the 59 lncRNAs, for which we already designed qPCR primers, we determined if they were preferentially localized in the nucleus. Fractionation procedures of L428, L1236, and L540 cell lines were validated by qPCR for six transcripts with a known subcellular localization. This revealed the expected enrichment of
RPPH1, DANCR, and MT-TK transcripts in the cytoplasmic fractions and of MIAT1, ANRIL, and XIST transcripts in the nuclear fractions (Figure 4, A and B). Consistent with a cis-regulatory role, LINC00461 and FLJ42351 showed enrichment in the nuclear fractions and depletion in the cytoplasmic fractions. Analysis of the putative cis-regulated protein-coding genes by RT-qPCR confirmed the inverse expression pattern between LINC00461 and the nearby upstream protein-coding gene, MEF2C (Supplemental Figure S2C). For FLJ42351, we could confirm the comparable differential expression patterns for this lncRNA and the neighboring protein-coding gene SLC20A1.

\section{Discussion}

In this study, we showed a dynamic regulation of lncRNA expression during the transition of $\mathrm{B}$ cells through the $\mathrm{GC}$ (ie, from naive B cell to GC-B cell, resulting in memory B cells). Furthermore, the comparison of cHL cell lines with GC-B cells revealed a significant differential expression pattern for a considerable number of lncRNAs. This indicates that lncRNAs play a role in normal B-cell maturation and that deregulated lncRNA expression is a prominent feature of HL.

We showed that the levels of 401 probes corresponding to 251 lncRNA loci change during the transition from naive to GC-B cells and reverse to levels comparable to naive B cells once the cells differentiate to memory B cells. Recently, 
Table 2 Table of Putative Cis-Regulated IncRNA-mRNA Pairs Identified in the Transcriptome Comparisons of the cHL Cell Lines and GC-B Cells

\begin{tabular}{|c|c|c|c|c|c|c|c|}
\hline \multirow[b]{2}{*}{ Chr } & \multicolumn{2}{|l|}{ IncRNA } & \multicolumn{2}{|l|}{ mRNA } & \multicolumn{3}{|c|}{ IncRNA to mRNA } \\
\hline & Probe name & Transcript(s) & Probe name & Gene symbol & Distance* $^{*}$ & Orientation & Direction $^{\dagger}$ \\
\hline 1 & CUST_4772_PI427622066 & TCONS_12_00001761,-2, -5 & A_23_P34915 & ATF3 & -44 & Tail-to-tail & Up/up \\
\hline 1 & CUST_4772_PI427622066 & TCONS_L2_00001761, $-2,-5$ & A_23_P160720 & BATF3 & 22 & Sense & Up/up \\
\hline 1 & CUST_4751_PI427622066 & TCONS_00001808 11 & A_23_P160720 & BATF3 & 54 & Sense & Up/up \\
\hline 2 & CUST_9360_PI427622066 & TCONS_00002672/ & A_23_P165657 & SLC20A1 & 19 & Head-to-head & Up/up \\
\hline
\end{tabular}

2 CUST_10333_PI427622066 2 CUST_8107_PI427622066

2 CUST_8408_PI427622066

2 CUST 6868 PI427622066

3 CUST_11860_PI427622066

3 A_33_P3368771

4 CUST_19108_PI427622066

4 CUST_12908_PI427622068

5 A_32_P127350

5 CUST_22952_PI427622066

6 CUST_20144_PI427622068

8 CUST_25512_PI427622068

9 A_33_P3574055

9 CUST_34071_PI427622066

9 A_23_P216935

9 A_23_P216935

10 CUST_40617_PI427622066

11 CUST_42984_PI427622066

11 A_23_P127367

12 CUST_44640_PI427622066

12 CUST_45746_PI427622066

12 CUST_44512_PI427622066

13 CUST_38642_PI427622068

14 A_23_P25746

14 CUST_40389_PI427622068

14 CUST_39858_PI427622068

15 CUST_41903_PI427622068

15 CUST_52013_PI427622066

15 CUST_50180_PI427622066

16 A_24_P865226

16 A_24_P865226

16 CUST_54455_PI427622066

17 CUST_55786_PI427622066

17 CUST_46171_PI427622068

17 A_32_P19000

17 A_32_P19000

17 CUST_46165_PI427622068

17 CUST_56085_PI427622066

18

A_32_P150876
TCONS_00005231/

FLJ42351

TCONS_00003045

TCONS_00003301

TCONS_L2_00014653

TCONS_00004118/

TCONS_00003184

TCONS_00006507

NR_026991.1/H1FX-AS1

TCONS_00009180 2

TCONS_00008872

NR_024384/LINC00461

TCONS_00011070

TCONS_12_00024170-2

TCONS_00014599,-600/

TCONS_00015212

TCONS_12_00028716, $-18 \sim 22$

TCONS_00015807

NR_026677.1/MIR600HG

NR_026677.1/MIR600HG

TCONS_00017839

TCONS_00019374

NR_046412/P0LD4

TCONS_00020398

TCONS_00020260/

NR_110375.1 / THRIL

TCONS_00021299

TCONS_00021766

A_23_P56559 DHRS9

A_33_P3316928 PELI1

A_23_P68072 WDR54

A_33_P3401826 CMPK2

A_33_P3288023

NCKIPSD

A_23_P251377

A_24_P337334

A_24_P103264

A_23_P320739

A_24_P97825

A_32_P351968

A_24_P201064

A_33_P3348001

A_23_P124855

A_24_P252575

A_23_P252155

A_23_P161481

A_23_P24444

A_23_P323227

A_33_P3215647

A_32_P831181

A_23_P98930

A_24_P10137

NR_027263/ARHGAP5-AS1

TCONS_00022548

TCONS_00022357

A_33_P3386459

A_24_P273742

A_23_P357717

TCONS_l2_00008581, -583

TCONS_L2_00008800

TCONS_L2_00009507

NR_015396.1/CDIPT-AS1

NR_024370.1/CDIPT-AS1

TCONS_12_00010376 80

TCONS_00025420, -926

TCONS_L2_00010942, -3

NR_040071.1/TNRC6C-AS1

NR_040071.1/TNRC6C-AS1

TCONS_00025471

TCONS_12_00011403,

$-406 \sim 409,-412 \sim 416$,

$-685 \sim 687$

NR_015389/LINC00667
A_33_P3260614

A_33_P3229276

A_33_P3262799

A_33_P3294608

A_23_P10194

A_24_P193582

A_23_P141429

A_33_P3344648

A_33_P3302428

A_23_P101013

A_33_P3344648

A_24_P941441

A_24_P74064

ZFP161

PLCB2

MVP

DEF8

$\mathrm{ABI} 3$

TMC6

GNA13
C3orf37

DCTD

UGT8

MEF2C

CCDC69

HLA-DMB

PPP1R3B

LOC100130458 -49

ZCCHC7

RABGAP1

STRBP

KIAA1274

DHCR7

PPP1CA

FAM113B

BRI3BP

C12orf35

C13orf15

ARHGAP5

TTC9

TCL1A

ZSCAN2

ZNF280D

SEZ6L2

LOC440461

TNRC6C

LOC440461

\section{$-5$}

6

12

18

$-51$

$-15$

13

$-6$

56

6

32

$-15$

$-26$

$-5$

15

$-42$

$-17$

47

$-13$

$-14$

33

$-36$

41

$-7$

$-5$

$-36$

13

25

$-17$

6

44

$-16$

$-6$

$-5$

4

11

37

44
Sense

Sense

Head-to-head

Sense

Down/down

Down/down

Sense

Sense

Sense

Sense

Sense

Sense

Down/up

Down/down

Tail-to-tail Down/down

Head-to-head Up/up

Head-to-head Down/down

Tail-to-tail Down/down

Tail-to-tail Down/down

Sense Down/down

Sense Down/down

Head-to-head Up/up

Sense

Sense

Down/down

Tail-to-tail Down/down

Tail-to-tail Down/down

Sense Down/down

Head-to-head Up/up

Sense Down/down

Head-to-head Down/down

Head-to-head Up/down

Sense

Down/up

Sense

Tail-to-tail

Down/down

Head-to-head Up/down

Sense

Sense

Antisense

Sense

Sense

Sense

Down/down

Down/down

Down/down Down/down Down/down Down/down

Tail-to-tail Down/down (table continues) 
Table 2 (continued)

\begin{tabular}{|c|c|c|c|c|c|c|c|}
\hline \multirow[b]{2}{*}{ Chr } & \multicolumn{2}{|l|}{ IncRNA } & \multicolumn{2}{|l|}{ mRNA } & \multicolumn{3}{|c|}{ IncRNA to mRNA } \\
\hline & Probe name & Transcript(s) & Probe name & Gene symbol & Distance* & Orientation & Direction $^{\dagger}$ \\
\hline 19 & A_33_P3685216 & NR_049780/ZNF837 & A_33_P3411427 & ZNF837 & 20 & Sense & Up/down \\
\hline 19 & CUST_59058_PI427622066 & $\begin{array}{l}\text { TCONS_00027367, -368, } \\
-759 / T C O N S \_00026816\end{array}$ & A_23_P90333 & ZNF404 & -28 & Sense & Down/down \\
\hline 19 & CUST_48777_PI427622068 & $\begin{array}{l}\text { TCONS_00026798, -99/ } \\
\text { TCONS_00027118 26 }\end{array}$ & A_33_P3418731 & ZNF542 & -14 & Sense & Down/down \\
\hline 19 & CUST_48637_PI427622068 & TCONS_L2_00012669 & A_23_P130444 & ZNF701 & -7 & Sense & Down/down \\
\hline 19 & A_33_P3646051 & NR_040027.1/ZNF790-AS1 & A_33_P3423401 & ZNF790 & 13 & Antisense & Down/down \\
\hline 19 & CUST_59260_PI427622066 & $\begin{array}{l}\text { TCONS_L2_00013339/ } \\
\text { TCONS_L2_00012668 }\end{array}$ & A_33_P3256868 & ZNF83 & 13 & Tail-to-tail & Down/down \\
\hline 20 & CUST_50118_PI427622068 & TCONS_00027871 & A_24_P91566 & BMP7 & -54 & Head-to-head & Down/down \\
\hline
\end{tabular}

The IncRNA-mRNA pairs that overlap with the putative cis-regulated IncRNA-mRNA pairs identified in the transcriptome comparisons of the normal B-cell subsets (Supplemental Table S2) are in bold.

*Probe to probe distance in $\mathrm{kb}$, positive distance means the IncRNA probe is upstream of the protein-coding gene, negative distance indicates the reverse.

${ }^{\dagger}$ The direction of the IncRNA is indicated first, the direction of the mRNA is indicated second. Direction is based on cHL cell lines compared with GC-B cells.

four studies analyzed lncRNA expression in B-cell populations. $^{22-25}$ Only one of the four studies also analyzed naive, memory, and germinal center B-cell populations isolated from tonsil. Consistent with our study, they showed that the IncRNA expression profiles of memory and naive $\mathrm{B}$ cells are similar and more distinct from GC-B cells. ${ }^{22}$ However, none of the studies provided overviews of the lncRNAs differentially expressed between similar B-cell subsets as used in our study, preventing a direct comparison to our results. At the mRNA level, we and others observed a similar pattern as we observed for lncRNAs (ie, similar
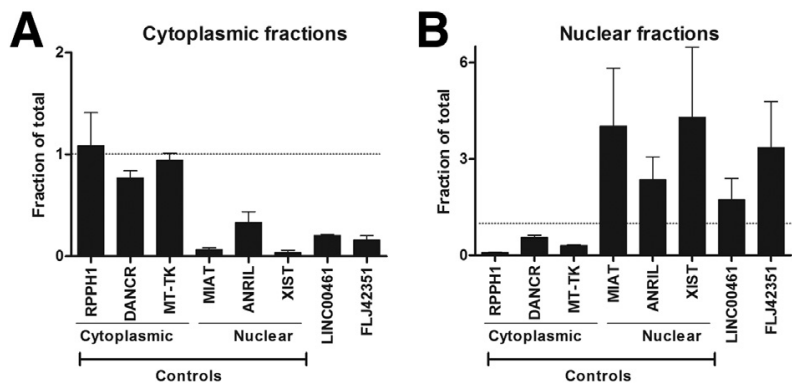

Figure 4 LINC00461 and FLJ42351 are nuclear enriched IncRNAs. As a validation for the isolation of cytoplasmic (A) and nuclear (B) fractions, the enrichment of three cytoplasmic control RNAs (RPPH1, DANCR, and MTTK) in the cytoplasmic fraction and three nuclear control (MIAT, ANRIL, and XIST) RNAs in the nuclear fraction were confirmed by quantitative RT-PCR. Analysis of LINC00461 and FLJ42351 shows a clear enrichment in the nuclear fraction and depletion in the cytoplasmic fraction. $\Delta \Delta \mathrm{Ct}$ values are shown with the average of the total fractions of L428, L1236, and L540 HL cell lines set to 1 (dashed line). 18S (cytoplasmic) and U3 (nuclear) were used for normalization of cytoplasmic and nuclear fractions, respectively. profiles for naive and memory $\mathrm{B}$ cells and significantly different profiles for GC-B cells). ${ }^{26}$ The NLP cell line DEV clustered with normal GC-B cells, and the cHL cell lines cluster in a sub-branch next to the GC-B cells for both the lncRNAs as well as the mRNA probes. This indicates that the expression profile of lymphocyte predominant cells of NLPHL more closely resembles that of GC-B cells than that of the HRS cells of cHL. These results are consistent with previous gene expression studies showing a loss-of-B-cell phenotype in HRS cells ${ }^{27}$ and intermediate expression patterns in lymphocyte predominant cells. ${ }^{28}$

A direct comparison of cHL cell lines with GC-B cells revealed significant differences for 639 probes (475 lncRNA loci). Almost $75 \%$ of these lncRNAs were down-regulated in the cHL cell lines, whereas the percentage of up-regulated and down-regulated mRNAs was similar. This suggests that lncRNAs might, similar to miRNAs, ${ }^{29}$ show a global downregulation in cancer. Previous studies comparing the expression profiles of microdissected primary HRS cells and cHL cell lines to GC-B cells showed a considerable overlap between the differentially expressed genes in primary HRS cells and cHL cell lines. ${ }^{27,30}$ However, a subset of the genes differentially expressed in primary HRS cells, including chemotaxis and surface receptor signaling related genes, were not differentially expressed in the cell lines. ${ }^{27,30}$ This indicates that it is likely that the lncRNA expression profile of the HL cell lines will also not fully reflect the lncRNA profile of primary HRS cells.

Three of the up-regulated IncRNAs (ie, LINC00116, LINC00461, and FLJ42351) were studied in more detail, 
including expression analysis in primary $\mathrm{cHL}$ tissues. RNAFISH for LINC00116 revealed a remarkable specific expression pattern, with fluorescent signals being restricted to the HRS cells. The RT-qPCR across normal B-cell subsets and lymphoma cell lines showed a similar pattern with a preferential expression in HL cells. TCGA data did not show a preferential expression pattern across multiple other types of cancer. LINC00116 has three known transcript variants, two short variants and one long variant. The longer transcript (LINC00116-001) might encode for a 138 amino acid uncharacterized protein (http://www.uniprot.org/uniprot; accession number Q8NCU8), whereas the shorter variants represent true lncRNA transcripts without protein-coding potential. The expression pattern of the probes present on our array pointed to a predominant expression of shorter isoforms (LINC00116-002 and LINC00116-003) (Supplemental Figure S3). Moreover, the probes for RNA-FISH and the primers used for the qPCR were also designed on the basis of these short noncoding transcript isoforms. Thus, our data support an HRS cell-specific expression pattern of one or both of the shorter LINC00116 transcript variants.

LINC00461 showed a highly specific expression pattern in the HRS cells by RNA-FISH. We also noticed enhanced expression of LINC00461 in HL, DLBCL, and LCL cell lines. Analysis of the TCGA data revealed expression only in lowgrade gliomas and not in DLBCL tissues. The difference between the qPCR and TCGA results for DLBCL may be related to differences that sometimes can be observed between cell lines and primary tissues. The LINC00461 locus contains several isoforms of which the array probe, the RT-qPCR primer set, and the FISH probe set all detect both the 3.6-kb (NR_024384) and the 3.4-kb (NR_024383) isoforms (Supplemental Figure S3). The last exon of both lncRNA isoforms contains the pre-miR-9-2 sequence. Whether this IncRNA, besides serving as the primary transcript of miR-9, also has other functions has yet to be determined. It has been reported that miR-9 expression is increased in $\mathrm{HL}$ and glioma and that inhibition of miR-9 inhibits growth of HL cell lines in a xenograft mouse model. ${ }^{31-34}$ The expression pattern of the nuclear enriched LINC00461 showed an inverse correlation with the expression of the nearby upstream protein-coding gene, $M E F 2 C$. MEF2C is essential for B-cell proliferation and survival in response to BCR stimulation in vitro. ${ }^{35}$ In vivo, MEF2C loss results in reduced antibody responses to T-cell-dependent antigens and impaired GC formation. ${ }^{35,36}$ Studies in rats have shown that MEF2C can stimulate expression of the miR-9-2 locus by binding to a highly conserved site in the promoter of the miR-9-2 host gene. ${ }^{37}$ On the other hand, MEF2C is a predicted miR-9 target via a broadly conserved 8 -mer site in the $3^{\prime}$ untranslated region. How LINC00461 and $\mathrm{MEF} 2 \mathrm{C}$ regulate each other's expression and to what extent this is relevant for $\mathrm{cHL}$ remains to be investigated.

FLJ42351, the third lncRNA studied in more detail, also showed a remarkable HRS cell-specific expression in primary HL tissues. qPCR confirmed expression in HL cell lines and showed in addition expression in DLBCL and LCL cell lines. TCGA RNA-seq data did not show substantial expression in other types of cancer tissues. The expression pattern of FLJ42351 is similar to that of the immediately downstream phosphate transporter SLC20A1. Consistent with a cis-regulatory role of FLJ42351 we observed a preferential nuclear localization in the cHL cell lines. SLC20A1 knockdown in Hela and hepatic cell lines reduced cell proliferation, ${ }^{38}$ and SLC20A1 knockout mice showed increased apoptosis rates in erythroid cells. ${ }^{39} \mathrm{We}$ previously identified FLJ42351 as a MYC-induced transcript in P493-6 cells. ${ }^{17}$ In support of a putative cis-regulatory role of this lncRNA, we also identified SLC20A1 as a positively correlating neighboring gene of FLJ42351 in our MYC-regulated lncRNA study. Thus, in summary, FLJ42351 is a HRS cell-expressed IncRNA whose expression pattern is similar to that of the neighboring protein-coding gene SLC20Al, which is known to have important functions in cell growth.

Despite several attempts, we were not able to efficiently knock down LINC00461 and FLJ42351 with siRNA- and lentiviral shRNA-based approaches. This precluded a further analysis to provide more definitive proof that these two lncRNAs can regulate the expression of the neighboring genes. Interestingly, both lncRNAs do have a DNase I hypersensitivity site, which is indicative of regulatory sequences (ie, approximately $15 \mathrm{~kb}$ upstream of the transcriptional start site of LINC00461 in both L1236 and L428 cell lines ${ }^{40}$ and approximately $45 \mathrm{~kb}$ downstream of the FLJ42351 transcriptional start site in L1236).

In summary, our comprehensive expression analysis shows dynamic regulation of lncRNA expression during the GC transition of B cells and a widely deregulated expression in HL. These IncRNAs, together with the putative cis-acting data, provide a valuable source for further studies aiming at understanding the role of lncRNAs in normal B-cell biology and in the pathogenesis of HL.

\section{Acknowledgments}

We thank Klaas Sjollema (University Medical Center Groningen) for providing help with the imaging.

M.M.T., G.K., J.S., and D.d.J. performed research; M.M.T., M.W., M.T., P.M., L.V., A.D., K.K., and J.K. analyzed data; F.G.M.K. contributed essential samples; and M.M.T., K.K., A.v.d.B., and J.K. designed the study and wrote the manuscript.

\section{Supplemental Data}

Supplemental material for this article can be found at http://dx.doi.org/10.1016/j.ajpath.2016.05.011.

\section{References}

1. Hjalgrim H, Engels EA: Infectious aetiology of Hodgkin and nonHodgkin lymphomas: a review of the epidemiological evidence. J Intern Med 2008, 264:537-548 
2. Schwering I, Brauninger A, Klein U, Jungnickel B, Tinguely M, Diehl V, Hansmann ML, Dalla-Favera R, Rajewsky K, Kuppers R: Loss of the B-lineage-specific gene expression program in Hodgkin and Reed-Sternberg cells of Hodgkin lymphoma. Blood 2003, 101: $1505-1512$

3. Hertel CB, Zhou XG, Hamilton-Dutoit SJ, Junker S: Loss of B cell identity correlates with loss of B cell-specific transcription factors in Hodgkin/Reed-Sternberg cells of classical Hodgkin lymphoma. Oncogene 2002, 21:4908-4920

4. Tsai HK, Mauch PM: Nodular lymphocyte-predominant hodgkin lymphoma. Semin Radiat Oncol 2007, 17:184-189

5. Kuppers R: B cells under influence: transformation of B cells by Epstein-Barr virus. Nat Rev Immunol 2003, 3:801-812

6. Jost PJ, Ruland J: Aberrant NF-kappaB signaling in lymphoma: mechanisms, consequences, and therapeutic implications. Blood 2007, 109:2700-2707

7. Plattel W, Kluiver J, Diepstra A, Visser L, van den Berg A: The role of microRNAs in Hodgkin's lymphoma. Edited by Lawrie CH: MicroRNAs in Medicine, ch26. Hoboken, NJ: John Wiley \& Son, Inc., 2013, pp 435-447

8. Derrien T, Johnson R, Bussotti G, Tanzer A, Djebali S, Tilgner H, Guernec G, Martin D, Merkel A, Knowles DG, Lagarde J, Veeravalli L, Ruan X, Ruan Y, Lassmann T, Carninci P, Brown JB, Lipovich L, Gonzalez JM, Thomas M, Davis CA, Shiekhattar R, Gingeras TR, Hubbard TJ, Notredame C, Harrow J, Guigo R: The GENCODE v7 catalog of human long noncoding RNAs: analysis of their gene structure, evolution, and expression. Genome Res 2012, 22:1775-1789

9. Ma L, Bajic VB, Zhang Z: On the classification of long non-coding RNAs. RNA Biol 2013, 10:925-933

10. Orom UA, Derrien T, Beringer M, Gumireddy K, Gardini A, Bussotti G, Lai F, Zytnicki M, Notredame C, Huang Q, Guigo R, Shiekhattar R: Long noncoding RNAs with enhancer-like function in human cells. Cell 2010, 143:46-58

11. Nagano T, Mitchell JA, Sanz LA, Pauler FM, Ferguson-Smith AC, Feil R, Fraser P: The Air noncoding RNA epigenetically silences transcription by targeting G9a to chromatin. Science 2008, 322: $1717-1720$

12. Guil S, Esteller M: Cis-acting noncoding RNAs: friends and foes. Nat Struct Mol Biol 2012, 19:1068-1075

13. Kung JT, Colognori D, Lee JT: Long noncoding RNAs: past, present, and future. Genetics 2013, 193:651-669

14. Prensner JR, Chinnaiyan AM: The emergence of 1 ncRNAs in cancer biology. Cancer Discov 2011, 1:391-407

15. Campo E, Swerdlow SH, Harris NL, Pileri S, Stein H, Jaffe ES: The 2008 WHO classification of lymphoid neoplasms and beyond: evolving concepts and practical applications. Blood 2011, 117:5019-5032

16. Tan LP, Wang M, Robertus JL, Schakel RN, Gibcus JH, Diepstra A, Harms G, Peh SC, Reijmers RM, Pals ST, Kroesen BJ, Kluin PM, Poppema S, van den Berg A: miRNA profiling of B-cell subsets: specific miRNA profile for germinal center B cells with variation between centroblasts and centrocytes. Lab Invest 2009, 89:708-716

17. Winkle M, van den Berg A, Tayari M, Sietzema J, Terpstra M, Kortman G, de Jong D, Visser L, Diepstra A, Kok K, Kluiver J: Long noncoding RNAs as a novel component of the Myc transcriptional network. FASEB J 2015, 29:2338-2346

18. Maggio EM, Van Den Berg A, Visser L, Diepstra A, Kluiver J, Emmens R, Poppema S: Common and differential chemokine expression patterns in rs cells of NLP, EBV positive and negative classical Hodgkin lymphomas. Int J Cancer 2002, 99:665-672

19. Blankenberg D, Von Kuster G, Coraor N, Ananda G, Lazarus R, Mangan M, Nekrutenko A, Taylor J: Galaxy: a web-based genome analysis tool for experimentalists. Curr Protoc Mol Biol 2010, Chapter 19. Unit 19.10.1-Unit 19.10.21

20. Goecks J, Nekrutenko A, Taylor J; Galaxy Team: Galaxy: a comprehensive approach for supporting accessible, reproducible, and transparent computational research in the life sciences. Genome Biol 2010, 11:R86
21. Cabili MN, Trapnell C, Goff L, Koziol M, Tazon-Vega B, Regev A, Rinn JL: Integrative annotation of human large intergenic noncoding RNAs reveals global properties and specific subclasses. Genes Dev 2011, 25:1915-1927

22. Petri A, Dybkaer K, Bogsted M, Thrue CA, Hagedorn PH, Schmitz A, Bodker JS, Johnsen HE, Kauppinen S: Long noncoding RNA expression during human B-cell development. PLoS One 2015, 10: e0138236

23. Ranzani V, Rossetti G, Panzeri I, Arrigoni A, Bonnal RJ, Curti S, Gruarin P, Provasi E, Sugliano E, Marconi M, De Francesco R, Geginat J, Bodega B, Abrignani S, Pagani M: The long intergenic noncoding RNA landscape of human lymphocytes highlights the regulation of $\mathrm{T}$ cell differentiation by linc-MAF-4. Nat Immunol 2015, 16:318-325

24. Bonnal RJ, Ranzani V, Arrigoni A, Curti S, Panzeri I, Gruarin P, Abrignani S, Rossetti G, Pagani M: De novo transcriptome profiling of highly purified human lymphocytes primary cells. Sci Data 2015, 2: 150051

25. Verma A, Jiang Y, Du W, Fairchild L, Melnick A, Elemento O: Transcriptome sequencing reveals thousands of novel long non-coding RNAs in B cell lymphoma. Genome Med 2015, 7:110

26. Klein U, Tu Y, Stolovitzky GA, Keller JL, Haddad J Jr, Miljkovic V, Cattoretti G, Califano A, Dalla-Favera R: Transcriptional analysis of the B cell germinal center reaction. Proc Natl Acad Sci U S A 2003, 100:2639-2644

27. Tiacci E, Doring C, Brune V, van Noesel CJ, Klapper W, Mechtersheimer G, Falini B, Kuppers R, Hansmann ML: Analyzing primary Hodgkin and Reed-Sternberg cells to capture the molecular and cellular pathogenesis of classical Hodgkin lymphoma. Blood 2012, 120:4609-4620

28. Brune V, Tiacci E, Pfeil I, Doring C, Eckerle S, van Noesel CJ, Klapper W, Falini B, von Heydebreck A, Metzler D, Brauninger A, Hansmann ML, Kuppers R: Origin and pathogenesis of nodular lymphocyte-predominant Hodgkin lymphoma as revealed by global gene expression analysis. J Exp Med 2008, 205:2251-2268

29. Lu J, Getz G, Miska EA, Alvarez-Saavedra E, Lamb J, Peck D, SweetCordero A, Ebert BL, Mak RH, Ferrando AA, Downing JR, Jacks T, Horvitz HR, Golub TR: MicroRNA expression profiles classify human cancers. Nature 2005, 435:834-838

30. Steidl C, Diepstra A, Lee T, Chan FC, Farinha P, Tan K, Telenius A, Barclay L, Shah SP, Connors JM, van den Berg A, Gascoyne RD: Gene expression profiling of microdissected Hodgkin Reed-Sternberg cells correlates with treatment outcome in classical Hodgkin lymphoma. Blood 2012, 120:3530-3540

31. Leucci E, Zriwil A, Gregersen LH, Jensen KT, Obad S, Bellan C, Leoncini L, Kauppinen S, Lund AH: Inhibition of miR-9 de-represses HuR and DICER1 and impairs Hodgkin lymphoma tumour outgrowth in vivo. Oncogene 2012, 31:5081-5089

32. Van Vlierberghe P, De Weer A, Mestdagh P, Feys T, De Preter K, De Paepe P, Lambein K, Vandesompele J, Van Roy N, Verhasselt B, Poppe B, Speleman F: Comparison of miRNA profiles of microdissected Hodgkin/Reed-Sternberg cells and Hodgkin cell lines versus CD77+ B-cells reveals a distinct subset of differentially expressed miRNAs. Br J Haematol 2009, 147:686-690

33. Nie K, Gomez M, Landgraf P, Garcia JF, Liu Y, Tan LH, Chadburn A, Tuschl T, Knowles DM, Tam W: MicroRNA-mediated downregulation of PRDM1/Blimp-1 in Hodgkin/Reed-Sternberg cells: a potential pathogenetic lesion in Hodgkin lymphomas. Am J Pathol 2008, 173:242-252

34. Nass D, Rosenwald S, Meiri E, Gilad S, Tabibian-Keissar H, Schlosberg A, Kuker H, Sion-Vardy N, Tobar A, Kharenko O, Sitbon E, Lithwick Yanai G, Elyakim E, Cholakh H, Gibori H, Spector Y, Bentwich Z, Barshack I, Rosenfeld N: MiR-92b and miR$9 / 9 *$ are specifically expressed in brain primary tumors and can be used to differentiate primary from metastatic brain tumors. Brain Pathol 2009, 19:375-383

35. Wilker PR, Kohyama M, Sandau MM, Albring JC, Nakagawa O, Schwarz JJ, Murphy KM: Transcription factor Mef2c is required for B 
cell proliferation and survival after antigen receptor stimulation. Nat Immunol 2008, 9:603-612

36. Homminga I, Pieters R, Langerak AW, de Rooi JJ, Stubbs A, Verstegen M, Vuerhard M, Buijs-Gladdines J, Kooi C, Klous P, van Vlierberghe P, Ferrando AA, Cayuela JM, Verhaaf B, Beverloo HB, Horstmann M, de Haas V, Wiekmeijer AS, Pike-Overzet K, Staal FJ, de Laat W, Soulier J, Sigaux F, Meijerink JP: Integrated transcript and genome analyses reveal NKX2-1 and MEF2C as potential oncogenes in T cell acute lymphoblastic leukemia. Cancer Cell 2011, 19:484-497

37. Davila JL, Goff LA, Ricupero CL, Camarillo C, Oni EN, Swerdel MR, Toro-Ramos AJ, Li J, Hart RP: A positive feedback mechanism that regulates expression of miR-9 during neurogenesis. PLoS One 2014, 9: e94348

38. Beck L, Leroy C, Salaun C, Margall-Ducos G, Desdouets C, Friedlander G: Identification of a novel function of PiT1 critical for cell proliferation and independent of its phosphate transport activity. J Biol Chem 2009, 284:31363-31374

39. Liu L, Sanchez-Bonilla M, Crouthamel M, Giachelli C, Keel S: Mice lacking the sodium-dependent phosphate import protein, PiT1 (SLC20A1), have a severe defect in terminal erythroid differentiation and early B cell development. Exp Hematol 2013, 41: $432-443 \mathrm{e} 7$

40. Kreher S, Bouhlel MA, Cauchy P, Lamprecht B, Li S, Grau M, Hummel F, Kochert K, Anagnostopoulos I, Johrens K, Hummel M, Hiscott J, Wenzel SS, Lenz P, Schneider M, Kuppers R, Scheidereit C, Giefing M, Siebert R, Rajewsky K, Lenz G, Cockerill PN, Janz M, Dorken B, Bonifer C, Mathas S: Mapping of transcription factor motifs in active chromatin identifies IRF5 as key regulator in classical Hodgkin lymphoma. Proc Natl Acad Sci U S A 2014, 111: E4513-E4522 